\title{
Towards a Medium-Independent Flow Sensor
}

\author{
Reyes Romero, Diego; Cubukcu, Ali; Urban, Gerald \\ IMTEK - Institut für Mikrosystemtechnik \\ Lehrstuhl Sensoren \\ Georges-Koehler-Allee 103 \\ D-79110 Freiburg / Deutschland
}

\begin{abstract}
We have successfully developed a low power two-dimensional calorimetric flow sensor in order to measure the wind speed on a blimp, which can also be used for measuring thermal properties of fluids. It features a central heater-thermistor pair surrounded by four additional germanium thermistors, which allow a temperature sensing resolution under $1 \mathrm{mK}$. Flow measurements strongly depend on the fluid's thermal conductivity $(\kappa)$, specific heat $\left(c_{p}\right)$ and density $(\rho)$. Since variations on these properties may result in a wrong flow rate measurement, recalibration is needed for different fluids. In this paper we discuss the effect of the thermal and physical properties of the fluid on the sensor's response. With this, we aim to develop a self-calibration method for the sensor. Simulations suggest that the frequency response of the temperatures at the thermistors exhibits a low-pass behavior. In this paper, we present experimental results, which suggest that the frequency response is not affected by the fluid's flow rate.
\end{abstract}

\section{Introduction}

At IMTEK, we have succesfully designed a micromachined calorimetric flow sensor, whose main application is to provide wind speed information in order to navigate a blimp. A typical calorimetric flow sensor is composed of a heating element surrounded by temperature sensors [1],[2],[3]. As a fluid passes by, it carries some heat from the heater to the temperature sensors, the temperature difference between them is measured. The relation between velocity and this temperature difference is linear for a larger range than it is for other types of flow sensors [4]. Many commercial devices based on this principle have been available in the market for several years. However, since the amount of transported heat depends on the fluid thermal properties, these type of sensors have to be calibrated according to the fluid. Our goal is to overcome this limitation. Some studies [2],[3] have shown that the thermal properties of the fluid have a unique effect on the sensor's frequency response. In this paper, we present the measured frequency response of our sensor. This response shows negligible independency from the fluid's state of motion, which will allow us to develop new calibration techniques, thereby increasing the versatility of the sensor.

\section{Sensor Description}

Many calorimetric flow sensors [2],[3],[4],[5] are composed of a single heating element surrounded by temperature sensing elements, e.g., thermopiles or thermistors, which detect the temperature variations due to the combined effect of the heater and the flowing medium. Our sensor also features a central thermistor, which is laid within the heating element as shown in Figure 1. Therefore, it is possible to measure the temperature at the heater and study its relationship with the temperatures at other points of the sensor. The heater is composed of four chromium structures. Four additional thermistors are placed concentrically around the heater. The thermistors were fabricated with germanium, hence it is possible to achieve a resolution under $1 \mathrm{mK}$ and a temperature coefficient of resistance around $2 \% / \mathrm{K}$ [1]. All these elements are placed on a $1.4 \mu \mathrm{m}$ thick membrane, which has a low thermal conductivity, thus the power drawn by the sensor is kept at a low value. This membrane has a circular shape with a diameter of $1 \mathrm{~mm}$. See [6] for details regarding the fabrication process. 


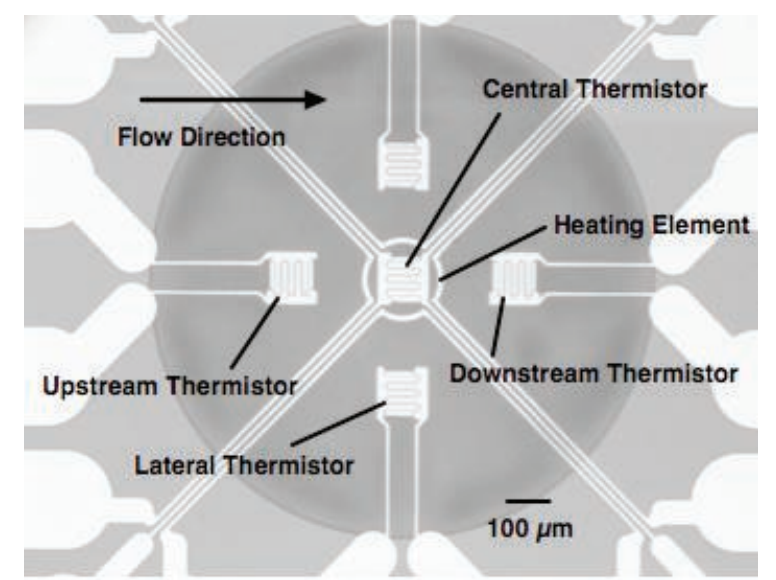

Figure 1. Top view of the sensor over the membrane of circular shape. Flow is meant to occur over the top face of the sensor. Four heaters lie around the central thermistor. Flow direction is parallel to one of the sensor's major axes.

\section{Experimental Setup}

The sensor is placed inside a standard wind channel (Testo) whose inner diameter is $10 \mathrm{~cm}$. Air flows inside the channel at different velocities $(1,3$, and $5 \mathrm{~m} / \mathrm{s})$ at room temperature. Given the dimensions of the channel, a nearly flat laminar flow profile is achieved at the point where the sensor is located. The flow direction is parallel to one of the major axes of the sensor as shown in the Figure 1. Thus, we can call the thermistors by its location with respect to the central thermistor as downstream (d), upstream ( $u$ ) and lateral (I) thermistors. The heater is operated with AC voltage at an average power of $0.5 \mathrm{~mW}$. The resistance change of the thermistors is then measured with a transresistance amplifier and the resulting voltage is digitalized and acquired by a computer. The temperature of the five thermistors is then recorded for input voltage frequencies between 0.5 and $100 \mathrm{~Hz}$. The oscillation frequency of the power signal is twice as large as the frequency of the voltage, therefore the frequency of the temperature signals vary between 1 and $200 \mathrm{~Hz}$. In this paper, $f$ will represent the frequency of the temperature signals.

Since our sensor features a central thermistor next to the heater, its temperature signal is therefore used as reference signal for the temperature in the other thermistors. Although the second harmonic of the input voltage and the temperature of the central thermistor are not expected to be exactly in phase, it can be assumed that this phase shift is rather small. By considering this, we define the following set of signals:

$$
\left\{h_{d}(t)=\frac{T_{d}(t)}{T_{h}(t)}, h_{u}(t)=\frac{T_{u}(t)}{T_{h}(t)}, h_{l}(t)=\frac{T_{l}(t)}{T_{h}(t)}\right\}
$$

Where $T_{d}, T_{u}, T_{l}$ and $T_{h}$ are the AC components of the temperature measured by the downstream, upstream, lateral and central thermistors respectively.

\section{Results}

From the data collected as described in the previous section, the amplitude and the phase of the signals in (1) are calculated. In order to achieve higher accuracy, the Fast Fourier Transform of the raw data was first filtered offline. We used a simple window-filter centered at the excitation frequency (f), this filter has a bandwidth of $0.2 f$. Then, the filtered signals in time domain were used in order to calculate the relations in (1). Thus, the phase of $h_{d}$ is the phase shift between $T_{d}$ and $T_{h}$, and its amplitude represents the ratio between the temperature oscillation amplitudes at the downstream and the central thermistor. 


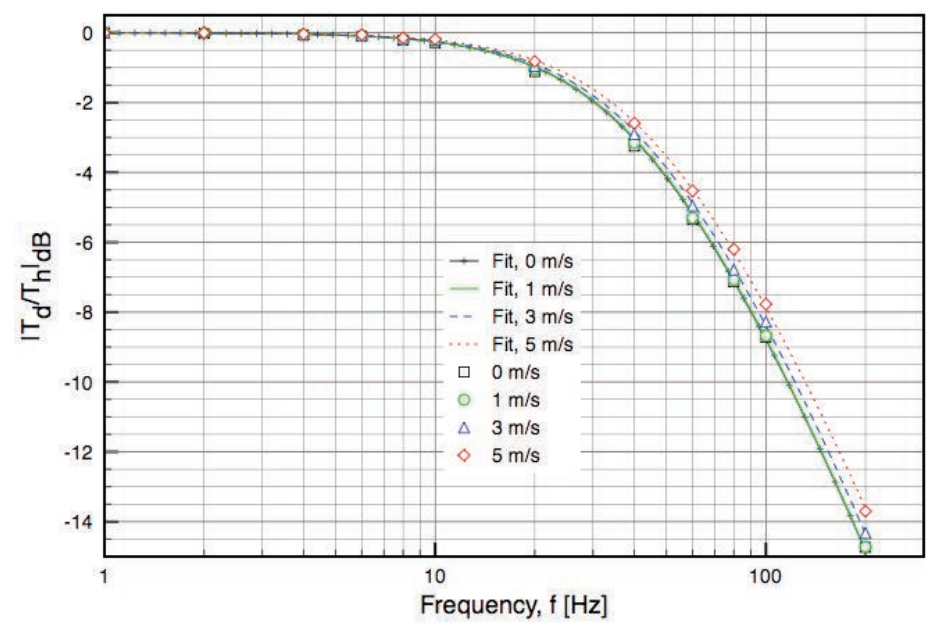

Figure 2. Normalized amplitude of the ratio $\left(h_{d}\right)$ between the temperature oscillation at the downstream thermistor $\left(T_{d}\right)$ and the temperature oscillation at the central thermistor $\left(T_{h}\right)$ in $\mathrm{dB}$ for several flow speeds. The normalization factor is the amplitude value of $h_{d}$ at $1 \mathrm{~Hz}$. The dots correspond to the experimental results whereas the curves correspond to the least squares fits to a second order system frequency response.

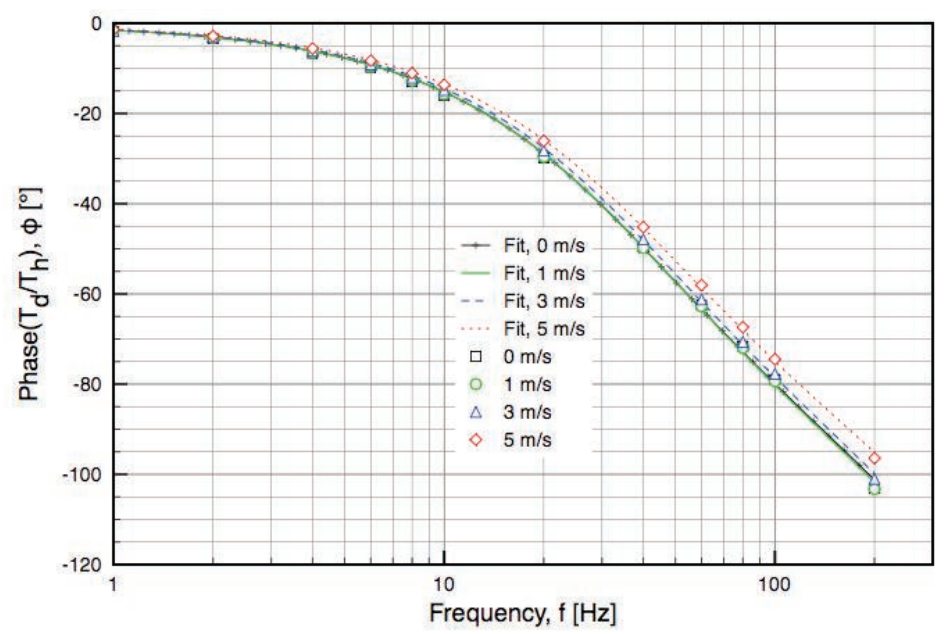

Figure 3. Phase shift between the temperature at the downstream thermistor $\left(T_{d}\right)$ and the temperature at the central thermistor $\left(T_{h}\right)$. The dots correspond to the experimental results whereas the curves correspond to the least squares fits to a second order system frequency response.

Figure 2 and Figure 3 depict the normalized amplitude (in $\mathrm{dB}$ ) and the phase of $h_{d}$ respectively for different flow rates. For the range of frequencies beyond $50 \mathrm{~Hz}$, the amplitude of $h_{d}$ seems to decay at a rate of $20 \mathrm{~dB} / \mathrm{dec}$, which fits well a first order RC system response. This result seems to concur with the simulation data presented in [7], which suggests that the sensor behaves akin to an RC system showing a low-pass response.

However, as Figure 3 shows, the phase shift is not asymptotically approaching $90^{\circ}$ as a first order system does. On the contrary, the phase shift goes beyond this limit suggesting a higher order behavior. Thus, we have performed, for each flow rate, a least squares fitting technique against a second order degree response over the empirical values of $h_{d}=\left|h_{d}\right| \exp \left(j \cdot \varphi_{d}\right)$, where $\left|h_{d}\right|$ is the amplitude and $\varphi_{d}$ is the phase shift. The results of these fittings are also depicted in Figure 2 and Figure 3 as smooth curves. For all four flow rates, the coefficient of determination $\left(R^{2}\right)$ is greater than 0.99 . Hence, a second order linear system seems to model correctly the sensor's response for frequencies up to $200 \mathrm{~Hz}$. The first pole remains close to $40 \mathrm{~Hz}$ for all flow rates, whereas the second pole lies on a frequency more than 10 times larger. Therefore, the amplitude of $h_{d}$ shows an unaltered first order dominant characteristic for all flow velocities.

This result suggests that the frequency response of the normalized amplitude and the phase shift seems to remain the same regardless the fluid's flow rate. This behavior could be explained by considering the equation describing the heat transport by conduction and forced convection in a one-dimensional, linear, homogeneous, isotropic medium [8]: 


$$
\frac{\rho c_{p}}{\kappa}\left(\frac{\partial T}{\partial t}+u_{x} \frac{\partial T}{\partial x}\right)=\frac{\partial^{2} T}{\partial x^{2}}+\frac{\dot{q}}{\kappa} .
$$

Where $q, \rho, c_{p}, \kappa$ and $u_{x}$ are the heat generation, the density, the specific heat, the thermal conductivity and the fluid velocity respectively. Most of the heat transfer between the central heater and the surrounding thermistors occurs through the membrane and the lower layers of fluid where the velocity is close to zero. Then, it is possible to assume that most of the heat transfer takes place along the direction parallel to the sensor plane. Therefore, by neglecting the heat generation for the regions excluding the heater, equation (2) becomes separable, i.e., $T(x, t)=X(x) \cdot T(t)$, where $X(x)$ and $T(t)$ are independent functions. Since the flow rate $u_{x}$ is not a factor of the time derivative in (2), the time function $T(t)$ does not include any flow rate term. As a consequence, the flow rate is expected not to affect the normalized frequency response of the system, or, at least, to have a weaker effect on the normalized frequency response than the fluid thermal properties.

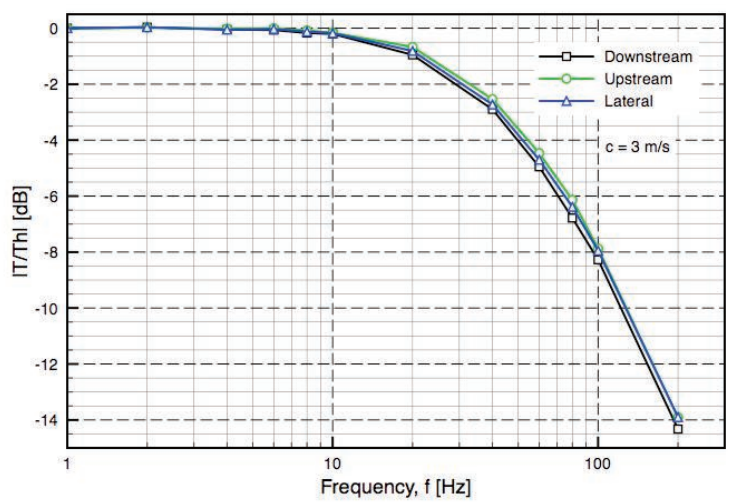

(a)

Figure 4. (a) Normalized amplitude of the ratios $\left(h_{i}, i=d, u, l\right)$ between the measured temperature oscillations at different thermistors $\left(T_{i}, i=d, u, l\right)$ and the temperature oscillation at the central thermistor $\left(T_{h}\right)$. The normalization factor is the amplitude value at $1 \mathrm{~Hz}$ at $3 \mathrm{~m} / \mathrm{s}$ flow speed. (b) Measured phase shift between the temperature at different thermistors $\left(T_{i}, i=d, u, l\right)$ and the temperature at the central thermistor $\left(T_{h}\right)$ at $3 \mathrm{~m} / \mathrm{s}$ flow speed.

Since the frequency response seems to be independent of the fluid's velocity, the upstream $\left(h_{u}\right)$ and lateral $\left(h_{l}\right)$ signals are expected to have a behavior similar to the downstream $\left(h_{d}\right)$ signal, which we have described above. Figure 4 shows the normalized amplitude (a) and phase shift (b) of $h_{d}, h_{u}$ and $h_{l}$ for a flow rate of $3 \mathrm{~m} / \mathrm{s}$. These curves exhibit almost no difference between them.

\section{Conclusion}

In summary, the normalized frequency response of the sensor seems to be a powerful tool in order to analyze the sensor's behavior. It proves to be independent of both the fluid's flow rate and the direction of the flow. This will be extremely helpful in order to devise a technique that makes our sensor mediumindependent.

Given the nature of our experimental setup, we have only conducted the experiments for air. Our future work foresees the use of several gases with different thermal properties in order to study their effect on the frequency response. Kuntner et al. [3] found that for liquids the amplitude of the thermal signals on a similar sensor is correlated to the thermal conductivity and the thermal diffusivity, whereas the phase shift remains constant for a single thermal diffusivity $\left(D=\kappa /\left(\rho . c_{p}\right)\right)$. Simulation results presented in [6] show that the specific heat capacity and density of the fluid are expected to have little influence on the temperature oscillation amplitude for a wide range of values. Additionally, according to [9], the thermal conductivity is the physical parameter that has the most dominant influence on the phase shift. Results in [3],[6],[9] prove that some simplifying assumptions can be made in order to extract the fluid's thermal properties from the frequency response. These assumptions are vital in order to design a simple algorithm that can be implemented in a small microprocessor. 


\section{Acknowledges}

We would like to thank Mr. Eugen Zernickel for conducting the experiment and exporting the data. We would also like to thank and recognize the financial support of the German Science Foundation (DFG) within the framework of the grant GRK 1103/1 Embedded Microsystems.

\section{References}

[1] A. S. Cubukcu, et al., "A 2D thermal flow sensor with sub-mW power consumption," Sens. Actuators A, vol. 163, pp. 449-456, 2010.

[2] R. Beigelbeck, et al., "Thermal Property Determination of Laminar-Flowing Fluids Utilizing the Frequency Response of a Calorimetric Flow Sensor," in IEEE Sensors 2008 Conf., Lecce, Italy, 2008, pp. 518-521.

[3] J. Kuntner, F. Kohl and B. Jakoby, "Simultaneous thermal conductivity and diffusivity sensing in liquids using a micromachined device," Sens. Actuators A, vol. 130-131, pp. 62-67, 2006.

[4] Elwenspoek, M., "Thermal Flow Micro Sensors," in Proc. CAS'99 International Semiconductor Conf., Sinaia, Romania, Vol. 2, pp. 423-435, Oct. 1999.

[5] F. Keplinger, et al., "Highly Sensitive Sensor for Flow Velocity and Flow Direction Measurement," in IEEE Sensors 2006 Conf., Daeugu, Korea, 2006, pp. 1436-1439.

[6] A. S. Cubukcu, G. A. Urban, "Simulation and Fabrication of a 2D-flow Sensor for simultaneous fluid characterization," in Proc. Eurosensors XXIII Conf., Lausanne, Switzerland, 2009, pp.

[7] A. S. Cubukcu, G. A. Urban, "Frequency Response of a 2D Flow and Thermal Property Sensor," in Proc. Eurosensors XXIII Conf., Lausanne, Switzerland, 2009, pp. 887-890.

[8] J. H. Lienhard, "Laminar and turbulent boundary layers," in A Heat Transfer Book, 3rd ed. Cambridge: Phlogiston Press, 2008, pp. 292-294.

[9] A. Cubukcu and G. Urban, "Simulation and fabrication of a 2d-flow sensor for thermal characterization of fluids and for flow speed measurements," in Proc. Transducers'09, Denver, USA, 2009, pp. 1126-1129. 